\title{
Correlação entre cálcio e insulina durante o teste de tolerância à glicose em ovelhas gestantes e não gestantes
}

\author{
[Calcium and insulin correlation during the glucose tolerance test \\ in pregnant non-pregnant ewes] \\ E. Schmitt ${ }^{1,2}$, A. Schneider ${ }^{1}$, M.A. Goulart ${ }^{1}$, E. Schwegler ${ }^{1}$, R.A. Pereira ${ }^{1}$, D.A.C. Hoffmann ${ }^{1}$, \\ M.S. Lopes ${ }^{1}$, L.T. Hax ${ }^{1}$, F.A.B. Del Pino ${ }^{1}$, M.N. Corrêa ${ }^{1}$ \\ ${ }^{1}$ NUPEEC, Núcleo de Pesquisa - Ensino e Extensão em Pecuaria - Universidade Federal de Pelotas, RS \\ ${ }^{2}$ EMBRAPA Rondônia, Porto Velho, RO
}

\begin{abstract}
RESUMO
Compararam-se as concentrações de cálcio, glicose e insulina em ovelhas gestantes e não gestantes submetidas ao teste de tolerância à glicose (TTG). Oito ovelhas gestantes (GG) e oito não gestantes (NG) foram submetidas ao TTG por meio da administração endovenosa de uma solução de glicose, na dose de $500 \mathrm{mg} / \mathrm{kg}$ de peso vivo. Amostras de sangue foram coletadas nos tempos de -15, zero, 15, 30, 45, 60, 90, 120, 150 e 180 minutos, sendo zero min o momento da infusão, para avaliação da concentração sanguínea de glicose, cálcio e insulina. Foi avaliada a taxa de metabolização de glicose e insulina, por meio do cálculo da área sob a curva (ASC). As concentrações de glicose, cálcio, insulina e a ASC da insulina não diferiram entre os grupos ( $\mathrm{P}>0,05)$, entretanto as três últimas ASCs da glicose foram mais elevados nas gestantes $(\mathrm{P}<0,05)$, que correspondem ao período entre 90 e $180 \mathrm{~min}$. O teste de correlação de Pearson revelou correlação negativa entre concentração de cálcio e insulina nas gestantes $(\mathrm{P}<0,05)$. Estes resultados indicam menor taxa de metabolização de glicose em ovelhas gestantes e uma possível relação de cálcio e insulina no terço final de gestação.
\end{abstract}

Palavras-chave: gestação, ovelha, teste de tolerância, glicose, insulina

\begin{abstract}
The aim of this study was to investigate glucose, insulin and calcium metabolism during the Glucose Tolerance Test (GTT) in pregnant or nonpregnant ewes. Eight pregnant (PG) and nonpregnant (NPG) ewes per group received a glucose endovenosus bolus containning $500 \mathrm{mg} / \mathrm{kg}$ in one minute. Blood samples were collected on $-15,0,15,30,45,60,90,120,150$ and 180 minutes interval $(0$ min injection). These samples were used to evaluate glucose, calcium, insulin concentration, as well as insulin and glucose metabolization rate, which were determined by calculating the Area Under the Curve (AUC). The calcium, glucose and insulin concentrations were not different between groups. However, the three last glucose ASCs were higher in the pregnant group and in this group calcium and insulin blood concentrations were negatively correlated.These results showed lower glucose tolerance in pregnant ewes and suggest a link between insulin and calcium in the late pregnancy.
\end{abstract}

Keywords: ewe, glucose tolerance test, insulin, pregnancy

\section{INTRODUÇÃO}

A gestação provoca diversas alterações no metabolismo materno, aumentando em aproximadamente $75 \%$ a demanda nutricional no final da gestação (Bauman e Currie, 1980). Esta

Recebido em 6 de junho de 2011

Aceito em 10 de maio de 2012

E-mail: schmitt.edu@gmail.com condição induz a adaptações no metabolismo da gestante, levando à maior ingestão calórica e ao aumento da resposta secretória de insulina (Brelje et al., 1993). Nesse período, cerca de $35 \%$ da glicose circulante da gestante são direcionadas para atender à demanda energética da unidade feto-placentária, sustentada pela redução progressiva e reversível da sensibilidade 
à insulina nos tecidos periféricos da gestante (Hay et al., 1983). Dessa forma, o feto adquire uma vantagem competitiva quanto à utilização de carboidratos, garantindo seu desenvolvimento final (Holness e Sugden, 1999).

Assim como no metabolismo dos carboidratos, a demanda de cálcio também atinge o seu pico no final da gestação devido à mineralização do esqueleto fetal, ocasionando adaptações no metabolismo da gestante (Brommage e Deluca, 1985). A participação desse mineral em importantes funções metabólicas vem sendo enfatizada por sua ação como segundo mensageiro celular em processos de exocitose dos sistemas endócrino, imune, nervoso e muscular (Oheim et al., 2006). A relevância desses sistemas para manutenção das funções vitais determina um rígido controle na homeostase do cálcio envolvendo os hormônios da paratireoide, a calcitonina e a vitamina D (Kovacs e Kronenberg, 1997). Apesar disso, a ocorrência de distúrbios, como a hipocalcemia, tem aumentado nas últimas décadas, principalmente em rebanhos leiteiros, pois estes estão correlacionados com a produção de leite (Goff, 2008).

Alguns autores têm correlacionado a manifestação dessa enfermidade com o balanço energético em ruminantes (Schlumbohm et al., 2003; Ingvartsen, 2006), e mesmo na década de 70 , já havia sido demonstrada em vacas leiteiras a relação da concentração de cálcio na diminuição da secreção de insulina (Blum et al., 1972). Recentemente essas relações foram mais bem exploradas, demonstrando a participação do cálcio nas diferentes fases de secreção de insulina pelas células $\beta$-pancreáticas (Walz, 2007) e no metabolismo de carboidratos (Schlumbohm et al., 2003; Rutter et al., 2006).

Técnicas como o teste de tolerância à glicose (TTG) têm sido empregadas em ruminantes para a determinação da capacidade de metabolização de glicose e a resposta na liberação pancreática de insulina (Schlumbohm et al., 2003; Regnault et al., 2004). Tais estudos demonstram importantes diferenças entre gestantes e não gestantes, principalmente no terço final de gestação (Regnault et al., 2004), além de correlacionarem níveis plasmáticos de cálcio na gestante com a taxa de metabolização de glicose (Schlumbohm et al., 1997; Schlumbohm et al., 2003). Com base nestas evidências, buscou-se comparar as taxas de metabolização de glicose e insulina, bem como sua correlação com a concentração sérica de cálcio total em ovelhas gestantes e não gestantes submetidas ao teste de tolerância à glicose.

\section{MATERIAL E MÉTODOS}

Foram utilizadas oito ovelhas entre 98 e 130 dias de gestação (GG; média de 54,85kg) e oito não gestantes (NG; média de 46,75kg), categorizadas conforme escore de condição corporal (ECC), de 1 (muito magra) a 5 (muito gorda). A distribuição segundo a condição reprodutiva e o escore foi: NG, cinco ovelhas com ECC 2 e três com ECC 2.5; GG, uma com ECC 2 e cinco com ECC 2.5 e duas com ECC 3. As ovelhas estavam sob mesmo regime alimentar de semiconfinamento, com fornecimento de $1,5 \%$ do peso vivo de concentrado contendo $15 \%$ de proteína bruta, $12 \%$ de matéria mineral, $1,5 \%$ de cálcio, $0,9 \%$ de fósforo, $2 \%$ de extrato etéreo e $13 \%$ de matéria fibrosa. Além do concentrado, as ovelhas permaneciam seis horas em pastagem de aveia (Avena sativa) e azevém (Lolium multiflorium).

Após um jejum alimentar de 12 horas, com livre acesso à água, as ovelhas foram submetidas durante um minuto à administração endovenosa de uma solução de glicose, na dose de $500 \mathrm{mg} / \mathrm{kg}$ de peso vivo (Regnault et al., 2004). O momento da administração da glicose foi considerado o momento zero, sendo realizadas coletas de sangue em -15, zero, 15, 30, 45, 60, 90, 120, 150 e 180 minutos da infusão de glicose. As coletas de sangue foram realizadas por punção da veia jugular, utilizando-se o sistema Vacutainer ${ }^{\circledR}$ (BD Diagnostics, São Paulo, Brasil), com um tubo contendo anti-coagulante (EDTA) e fluoreto de potássio, para avaliação da concentração de glicose, e outro sem anticoagulante, para avaliação da concentração de cálcio e insulina. As análises de cálcio total (Cálcio Liquiform, Labtest $\AA$, Lagoa Santa, Brazil, limite de detecção fotométrica $0,024 \mathrm{mg} / \mathrm{dL}$ ) e glicose (Glicose PAP Liquiform, Labtest ${ }^{\circledR}$, Lagoa Santa, Brazil, limite de detecção fotométrica $0,41 \mathrm{mg} / \mathrm{dL}$ ) foram realizadas pelo método colorimétrico, conforme as recomendações do fabricante, utilizando-se o espectrofotômetro 
Femto $650^{\circledR}$ (Femto Ind. e Com. de Instrumentos Ltda., São Paulo, Brazil). As amostras de insulina foram analisadas pelo método de eletroquimioluminescência, utilizando-se o Elecsys ${ }^{\circledR}$ Systems 2010 e metodologia conforme foi descrita pelo fabricante do Kit Insulina Roche $^{\circledR}$.

Para o cálculo da área sob a curva (ASC) da glicose e insulina foi utilizada a área do trapézio formado entre duas coletas subsequentes no gráfico (Regnault et al., 2004), considerando-se as alterações com relação ao nível basal de cada indivíduo (Área $=($ Valor Coleta $1-$ Valor Basal + Valor Coleta 2 - Valor Basal)*Intervalo entre coletas/2). Dessa forma, foram geradas nove ASCs para cada grupo, as quais foram comparadas dentro destes com seus respectivos períodos (ex.: ACS 1 gestantes vs. ASC 1 controle).

Os resultados, analisados utilizando-se o software SAS $^{\circledast}$ (SAS Institute Inc. Cary, NC, USA), foram apresentados como média \pm erropadrão da média. Foi utilizada análise de variância para medidas repetidas considerando-se como repetições o tempo de coleta, como unidade experimental a ovelha, como efeito randômico os animais dentro de seus respectivos grupos, e como efeito fixo os tratamentos. As variáveis-resposta foram: concentrações de glicose, cálcio e insulina, área sob a curva de glicose (ASCg) e área sob a curva de insulina (ASCi) nas comparações entre grupos e interações tempo versus grupos. Nas comparações entre médias, foram consideradas diferenças quando $\mathrm{P}<0,05$, segundo teste de Tukey-Kramer. Estimou-se, também, a correlação de Pearson entre as variáveis.

\section{RESULTADOS E DISCUSSÃO}

As concentrações médias de cálcio sérico total não diferiram $(\mathrm{P}>0,05)$ entre ovelhas gestantes $\mathrm{e}$ não gestantes, $8,52 \pm 0,18$ vs. $8,82 \pm 0,09 \mathrm{mg} / \mathrm{dL}$, durante todo o período experimental. Embora estejam entre 8,5 e $12,5 \mathrm{mg} / \mathrm{dL}$, considerados como padrões de referência para a espécie (Watt,
2006; El-Khodery et al., 2008), estes resultados diferem de estudos que demonstraram concentrações mais baixas de cálcio em ovelhas no terço final de gestação (Roubies, 2006). Ao longo das coletas, a concentração de cálcio variou nos dois grupos $(\mathrm{P}<0,05)$, possivelmente obedecendo a um padrão de regulação hormonal minuto a minuto (Fig. 1) exercido pelos hormônios da paratireoide (PTH), pela calcitonina e pela vitamina D (Kovacs et al., 1997). Essa dinâmica, similar nas gestantes e não gestantes, também explicaria a média geral de cálcio total, sem diferença entre grupos (Kovacs et al., 1997).

No entanto, a estimativa de correlação revelou que nas gestantes a concentração de cálcio correlacionou-se negativamente com a insulina $(\mathrm{P}<0,01)$, enquanto nas não gestantes a correlação indicou apenas uma tendência $(\mathrm{P}<0,07)$. Nesse contexto, foi demonstrado em humanos que a administração de insulina aumenta a excreção de cálcio e magnésio (Defronzo et al., 1976), assim como em pacientes portadores de diabetes tipo II com resistência à insulina (Lee et al., 2006). Este mecanismo ainda não está completamente esclarecido, mas sabe-se que a insulina aumenta a excreção desses minerais por mudanças nas trocas eletrolíticas no túbulo contorcido distal, elevando a retenção de sódio e potássio, importante para pacientes insulino-dependentes portadores de hipertensão (Lee et al., 2006). Esta ação da insulina pode ter relevância clínica na ocorrência de hipocalcemia e hipomagnesemia em ruminantes alimentados com alto nível de energia no pré-parto, já que o grau de adiposidade neste período está associado a maiores concentrações séricas de insulina, aumentando a resistência a este hormônio nos tecidos periféricos (Beever, 2006; Kerestes et al., 2009; Roche et al., 2009). Tal fato, somado à maior taxa de filtração glomerular na gestante (Baylis, 1994; Thornburg et al., 2000), poderia predispor à hipocalcemia e à hipomagnesemia, pela perda destes minerais via filtração renal (Defronzo et al., 1976). 

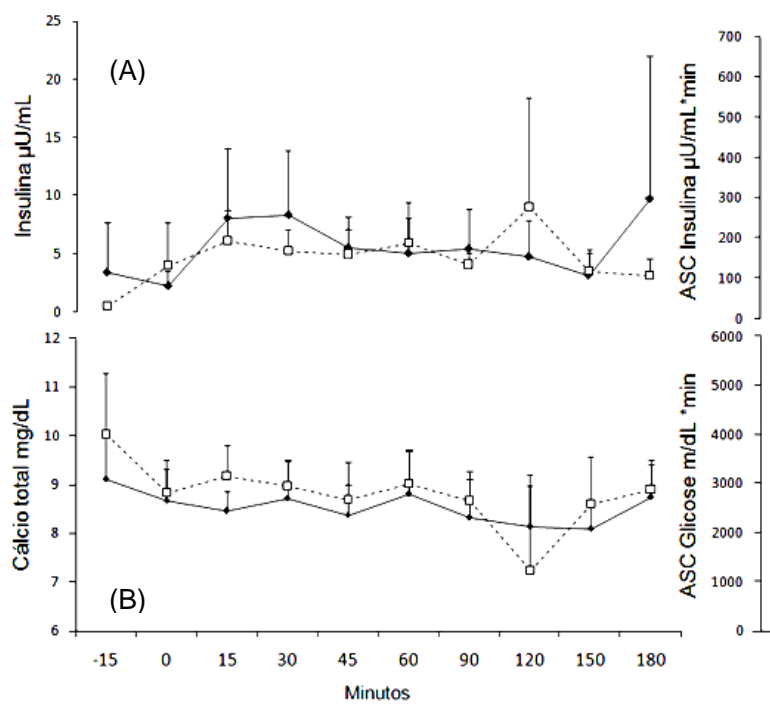

(C)
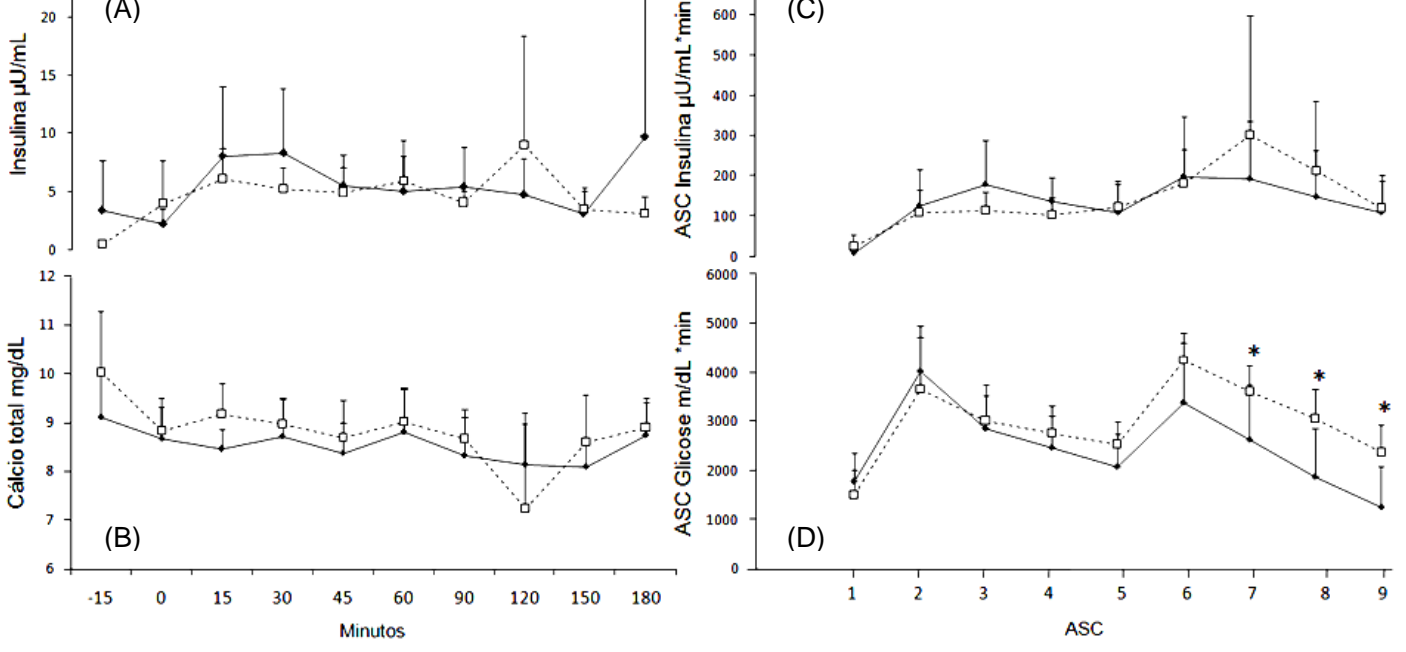

Figura 1. Concentrações sanguíneas de insulina $\left(\mu U \cdot \mathrm{mL}^{-1}\right)(\mathrm{A})$, cálcio total $\left(\mathrm{mg}_{\mathrm{g}} \mathrm{dL}^{-1}\right)(\mathrm{B})$ e área sob a curva de insulina (C) e glicose (D) de ovelhas gestantes ( $\square$ ) e não gestantes $(\diamond)$ durante o teste de tolerância à glicose. $* \mathrm{P}<0,05$, pelo teste de Tuckey.

Porém, a concentração de insulina e a ASCi não foram diferentes entre grupos (Fig. 1). Neste sentido, observou-se uma grande variação entre indivíduos do mesmo grupo (Fig. 1), para as variáveis insulina e glicose $(\mathrm{P}<0,01)$. Isto possivelmente tenha influenciado ovelhas gestantes e não gestantes a apresentarem um mesmo padrão $(\mathrm{P}>0,05)$, quanto às concentrações destas variáveis durante o TTG. A alta variação individual frente ao TTG vem sendo explicada pelos fatores epigenéticos que determinam a programação celular dos tecidos já na sua vida fetal de acordo com ambiente uterino a que são expostos (Youngson et al., 2008). Estes fatores, por sua vez, influenciam o metabolismo energético na vida adulta compondo importantes diferenças entre indivíduos, alterando padrões como a secreção de insulina e a responsividade frente a este hormônio nos tecidos periféricos (Husted et al., 2008).

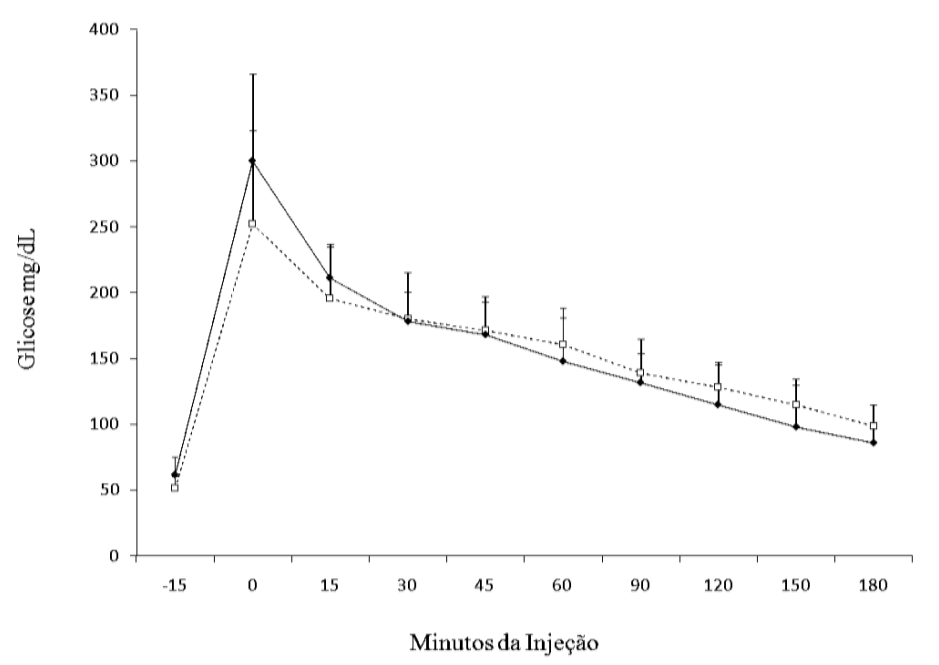

Figura 2. Concentrações sanguíneas de glicose $(\mathrm{mg} / \mathrm{dL})$ de ovelhas gestantes $(\square)$ e não gestantes $(\diamond)$ durante o teste de tolerância à glicose. 
Apesar da alta variação individual, as ASCs da glicose correspondentes aos períodos entre $90 \mathrm{e}$ 180min (ASC 7, 8 e 9) foram diferentes entre grupos, demonstrando um padrão de desaparecimento da glicose menor nas gestantes (Fig. 1). Estes resultados estão de acordo com estudos que demonstraram menor capacidade de metabolização da glicose em ovelhas no terço final de gestação, pela maior resistência dos tecidos periféricos à insulina (Schlumbohm, 1997; Regnault et al., 2004). Conforme a gestação avança em ovinos, a concentração materna de insulina, assim como a resposta da insulina a uma carga de glicose, é significativamente reduzida (Fowden, 1982). Apesar disso, as concentrações de glicose (Fig. 2) antes do TTG estavam dentro dos valores de referência observados por outros autores e considerados fisiológicos para ovelhas gestantes e não gestantes (Williams et al., 2004; Caldeira, 2005).

\section{CONCLUSÕES}

Ovelhas no final da gestação apresentam menor taxa de metabolização de glicose. Além disso, a correlação negativa entre cálcio e insulina nesta categoria sugere a participação da insulina na homeostase de cálcio no final da gestação.

\section{REFERÊNCIAS}

BAUMAN, D.E.; CURRIE, W.B. Partitioning of Nutrients During Pregnancy and Lactation: A Review of mechanisms involving homeostasis and homeorhesis. J. Dairy Sci., v.63, p.1514-1529, 1980.

BAYLIS, C. 2 Glomerular filtration and volume regulation in gravid animal models. Original Baillière's Clin. Obstetr. Gynecol., v.8, p.235-264, 1994.

BEEVER D.E. The impact of controlled nutrition during the dry period on dairy cow health, fertility and performance. Anim. Reprod. Scie., v.96, p.212-226, 2006.

BLUM, J.W.; WILSON, R.B.; KRONFELD, D.S. Plasma insulin concentrations in parturient cows. $J$. Dairy Sci., v.56, p.459-464, 1972.

BRELJE, T.C.; SCHARP, D.W.; LACY, P.E. et al. Effect of homologous placental lactogens, prolactins, and growth hormones on islet $\beta$-cell division and insulin secretion in rat, mouse, and human islets: implication for placental lactogen regulation of islet function during pregnancy. Endocrinology, v.132, p.879-887, 1993.
BROMMAGE, R.; DELUCA, H.F. Regulation of bone mineral loss during lactation. Am. J. Physiol., v.248, p.182-187, 1985.

CALDEIRA, R.M. Monitorização da adequação do plano alimentar e do estado nutricional em ovelhas. Rev. Port. Cienc. Vet., v.100, p.125-139, 2005.

DEFRONZO, R.A.; GOLDBERG, M.; AGUS, Z.S. The effects of glucose and insulin on renal electrolyte transport. J. Clin. Invest., v.58, p.83-90, 1976.

El-KHODERY, S.; El-BOSHY, M.; GAAFAR, K.; ELMASHAD, A. Hypocalcaemia in Ossimi Sheep Associated with Feeding on Beet Tops (Beta vulgaris). Turk. J. Vet. Anim. Sci., v.32, p.199-205, 2008.

FOWDEN, E.A.; ROSSDALE, P.D. Pancreatic $\beta$-cell function in the neonatal foal. J. Reprod. Fertil. Suppl., v.32 p.529-535, 1982.

GOFF, J.P. The monitoring, prevention, and treatment of milk fever and subclinical hypocalcemia in dairy cows. Vet. J., v.176, p.50-57, 2008.

HAY, JR. W.W.; SPARKS, J.W.; WILKENING, R.B. et al. Partition of maternal glucose production between conceptus and maternal tissues in sheep. Am. J. Physiol., v. 245, p.E347-E350, 1983.

HOLNESS, M.J.; SUGDEN, M.C. In late pregnancy insulin-dependent glucose transport/phosphorylation is selectively impaired and activation of glycogen synthase by insulin facilitated in skeletal muscles of 24-h starved rats. Diabetologia, v.42, p.802-811, 1999.

HUSTED, S.M.; NIELSEN, M.O.; BLACHE, D.; INGVARTSEN, K.L. Glucose homeostasis and metabolic adaptation in the pregnant and lactating sheep are affected by the level of nutrition previously provided during her late fetal life. Domest. Anim. Endocrinol., v.34, p.419-431, 2008.

INGVARTSEN, K.L. Feeding- and managementrelated diseases in the transition cow: Physiological adaptations around calving and strategies to reduce feeding-related diseases. Anim. Feed Scie. Technol., v.126, p.175-213, 2006.

KOVACS, C.S.; KRONENBERG, H.M. Maternalfetal calcium and bone metabolism during pregnancy, puerperium, and lactation. End. Rev., v.18, p.832-872, 1997.

KERESTES, M.; FAIGL, V.; KULCSÁR, M. et al. Periparturient insulin secretion and whole-body insulin responsiveness in dairy cows showing various forms of ketone pattern with or without puerperal metritis. Domest. Anim. Endocrinol., v.37, p.250-261, 2009.

LEE, C.T.; LIEN, Y.H.; LAI, L.W. et al. Increased renal calcium and magnesium transporter abundance in streptozotocin-induced diabetes mellitus. Kidney, v.69, p.1786-1791, 2006. 
OHEIM, M.; KIRCHHOFF. F.; STUHMER, W. Calcium microdomains in regulated exocytosis. Cell Calcium, v.40, p.423-439, 2006.

REGNAULT, T.R.H.; ODDY, H.V.; NANCARROW, C. et al. Glucose-stimulated insulin response in pregnant sheep following acute suppression of plasma non-esterified fatty acid concentrations. Rep. Biol. End., v.2, p.64, 2004.

ROCHE, J.R.; FRIGGENS, N.C.; KAY J.K. et al. Invited review: Body condition score and its association with dairy cow productivity, health, and welfare. J. Dairy Sci., v.92, p.5769-801, 2009.

ROUBIES, N.; PANOUSIS, N.; FYTIANOU, A. et al. Effects of Age and Reproductive Stage on Certain Serum Biochemical Parameters of Chios Sheep Under Greek Rearing Conditions. J. Vet. Med., v.53, p.277281, 2006.

RUTTER, G.A.; TSUBOI, T.; RAVIER, M.A. $\mathrm{Ca}^{2+}$ microdomains and the control of insulin secretion. Cell Calcium, v.40, p.539-551, 2006.

SCHLUMBOHM, C.; SPORLEDER, H.P.; GURTLER, H.; HARMEYER, J. The influence of insulin on metabolism of glucose, free fatty acids and glycerol in normo- and hypocalcemic ewes during different reproductive states. Deut. Tierarz. Wochen., v.104, p.359-365, 1997.
SCHLUMBOHM, C.; HARMEYER, J. Hypocalcemia reduces endogenous glucose production in hyperketonemic sheep. J. Dairy Sci., v.86, p.1953$1962,2003$.

THORNBURG, K.L.; JACOBSON, S.L.; GIRAUD, G.D.; MORTON M.J. Hemodynamic Changes in Pregnancy. Seminars in Perinatol., v.24, p.11-14, 2000.

WALZ, H.A.; WIERUP, N.; VIKMAN, J. et al. $\beta$-cell PDE3B regulates $\mathrm{Ca}^{2+}$-stimulated exocytosis of insulin. Cel. Signalling, v.19, p.1505-1513, 2007.

WATT, B.R. Hypocalcaemia in lactating drought fed ewes supplemented with recommended levels of calcium. Aust. J. Experim. Agricul., v.46, p.6-7, 2006.

WILLIAMS, C.C.; CALMES, K.J.; FERNANDEZ, J.M. et al. Glucose metabolism and insulin sensitivity in Gulf Coast Native and Suffolk ewes during late gestation and early lactation. Small Ruminant Res., v.54, p.167-171, 2004.

YOUNGSON, N.A.; WHITELAW, E. Transgenerational epigenetic effects. Annu. Rev. Genomics Hum Genet., v.9, p.233-257, 2008. 